\title{
Architecting Ubiquitous Communication and Collaborative Automation based Machine Network Systems for Flexible Manufacturing
}

DOI:

10.1109/JSYST.2019.2918542

\section{Document Version}

Accepted author manuscript

Link to publication record in Manchester Research Explorer

Citation for published version (APA):

Meng, Z., Wu, Z., \& Gray, J. (2019). Architecting Ubiquitous Communication and Collaborative Automation based Machine Network Systems for Flexible Manufacturing. IEEE Systems Journal.

https://doi.org/10.1109/JSYST.2019.2918542

\section{Published in:}

IEEE Systems Journal

\section{Citing this paper}

Please note that where the full-text provided on Manchester Research Explorer is the Author Accepted Manuscript or Proof version this may differ from the final Published version. If citing, it is advised that you check and use the publisher's definitive version.

\section{General rights}

Copyright and moral rights for the publications made accessible in the Research Explorer are retained by the authors and/or other copyright owners and it is a condition of accessing publications that users recognise and abide by the legal requirements associated with these rights.

\section{Takedown policy}

If you believe that this document breaches copyright please refer to the University of Manchester's Takedown Procedures [http://man.ac.uk/04Y6Bo] or contact uml.scholarlycommunications@manchester.ac.uk providing relevant details, so we can investigate your claim.

\section{OPEN ACCESS}




\title{
Architecting Ubiquitous Communication and Collaborative Automation based Machine Network Systems for Flexible Manufacturing
}

\author{
Zhaozong Meng, Member, IEEE, Zhipeng Wu, Senior Member, IEEE, John Gray
}

\begin{abstract}
Creative integration of smarter machines with new emerging Information and Communication Technologies (ICTs) in manufacturing industry allows collaborative automation between machines and promises greater production flexibility and product variability. The ubiquitous Machine-to-Machine (M2M) messaging, inter-machine understanding and system modeling are key enabling techniques to empower machine interoperability for Flexible Manufacturing Systems (FMS). However, the heterogeneities in machine part platforms, production strategies, and product variability keep the rich sensing and communication intensive sub-systems from seamless integration. By highlighting the specificities of industrial networks, this investigation aims to gain manufacturing flexibility by constructing a collaborative automation based industrial network from system and software perspective, focusing on: (1) the modeling of machine interactions in communication intensive industrial networks, (2) machine modularization and decentralized structure for production line scale efficiency, (3) ubiquitous messaging and understanding for machine interoperability, and (4) model-based management for application-level adaptation and flexibility. The presented technical solutions have been implemented in the PickNPack food manufacturing line, and the results demonstrate their feasibility.
\end{abstract}

Index Terms-Industrial networks, manufacturing flexibility, collaborative automation, ubiquitous M2M, interoperability.

\section{INTRODUCTION}

A CCORDING to Cisco, 500 billion devices are expected to be connected to the Internet by 2030. Each device includes sensors that collect data, interact with the environment, and communicate over a network, and the Internet of Things (IoT) is the network of these connected devices. The rapid progress of the IoT technologies and some new emerging ICTs are converging with a variety of application fields and continuously adding new features to them. They are driving the next industrial revolution by applying smarter machines, building connectivity between them, allowing them to communicate with and control one another for collaborative automation and intelligent optimization [1,2]. This futuristic concept is marked with some new terms by industrial professionals and communities, such as Industrial 4.0, Industrial IoT (IIoT), Smart Manufacturing, Manufacturing 2.0, and Cyber-Physical Systems (CPS). This new industrial computing paradigm is widely accepted as a solution to overcome the bottleneck of traditional software tools in dealing with complexity, dynamics and uncertainties of modern enterprise applications [3]. The future manufacturing industry will bring people benefits in many aspects, including minimum human involvement, highquality automation, efficient resource management, and Product Lifecycle Management (PLM). Therefore, it has been recognized as a new economic gain and a promising way to better quality of life [4].

The new industrial concept has created chances for flexible manufacturing that allows manufacturing systems to be automatically reconstructed to produce new product types by reconfiguring the functionality of the machines. The optimal modeling, data interoperability, inter-machine understanding, and real-time adaptation are the central issues to gain manufacturing flexibility. Apparently, the new opportunities of manufacturing industry are enabled by the emerging IoT techniques and novel computing paradigms.

Although manufacturing industrial networks share much in common with other IoT applications, IIoT scenarios distinguish from other IoT counterparts in the nature of machine interactions, where machines are tightly intertwined for interactive operations based on ubiquitous messaging and mutual understanding. The heterogeneities in machine part platforms, production strategies, and product variability keep the rich sensing and communication intensive sub-systems from seamless integration. The lack of well-suited production line scale strategies to enhance machine interoperability is identified as a major challenge to achieve higher level flexibility. This investigation is aimed to determine the specificities of manufacturing industrial network systems and provide customized IoT solutions to gain production line-level machine interoperability for flexible manufacturing.

The remainder of this paper is structured as follows: The enabling technologies, related studies and technological gaps are introduced in Section II. The customized solutions for manufacturing industry are presented in Section III. Then, the PickNPack practice as a case study is illustrated in Section IV. Finally, the lessons learned are given in Section V, conclusions are drawn and future perspectives are envisaged in Sections VI. The abbreviations in this article are summarized in TABLE I.

\section{BACKGROUND}

The emerging IoT techniques and novel computing paradigms can potentially improve the interoperability between the machines for collaborative automations by handling some intrinsic difficulties of industrial network systems. The 
TABLE I

LIST OF ABBREVIATIONS

\begin{tabular}{|c|c|c|c|}
\hline Abbreviation & Description & Abbreviation & Description \\
\hline ICT & Information and communication technology & AMQP & Advanced message queuing protocol \\
\hline M2M & Machine-to-machine & JMS & Java message service \\
\hline FMS & Flexible manufacturing system & REST & Representational state transfer \\
\hline IoT & Internet of things & COAP & Constrained application protocol \\
\hline IIoT & Industrial Internet of things & XMPP & Extensible messaging and presence protocol \\
\hline CPS & Cyber-physical system & ZMQ & Zero MQ \\
\hline PLM & Product lifecycle management & OPC UA & OPC unified architecture \\
\hline CCL & Connectivity and communication level & MRA & Manufacturing reference architecture \\
\hline MSL & Model and semantic level & SMLC & Smart manufacturing leadership coalition \\
\hline DKL & Data and knowledge level & DiRA & Reference architecture for discrete manufacturers \\
\hline P2P & Peer-to-peer & MTC & Machine type communication \\
\hline SDO & Standard development organization & NAPS & Naming, addressing, and profile server \\
\hline IEEE & Institute of electrical and electronics engineers & M2SP & M2M service platform architecture \\
\hline ETSI & European telecommunications standards institute & ARMU & Adaptive production management \\
\hline IETF & Internet engineering task force & SMN & Smart manufacturing network \\
\hline 3GPP & 3rd generation partnership project & KM/QM & Knowledge management/quality management \\
\hline OT/IT & Operation technology/information technology & UUID & Universal unique identifier \\
\hline CoBot & Collaborative robot & JSON & Javascript object notation \\
\hline WiFi & Wireless fidelity & FSM & Finite state machine \\
\hline 3G/4G/5G & $3^{\text {rd }}$ generation $/ 4^{\text {th }}$ generation $/ 5^{\text {th }}$ generation & GUI & Graphical user interface \\
\hline LTE & Long-term evolution & QAS & Quality assessment and sensing \\
\hline DDS & Direct distribution service & PLC & Programmable logic controller \\
\hline MQTT & Message queuing telemetry transport & OEE & Overall equipment effectiveness \\
\hline
\end{tabular}

ubiquitous data access and semantic understanding between devices in an IoT framework are key building blocks of interoperable machine networks for production flexibility and product variability. The blueprint is leveraged by the underlying techniques for inter-machine connectivity, communication and understanding.

\section{A. The Enabling Technologies}

A wide spectrum of technologies are involved to produce the efficiency and flexibility of IIoT applications, from low-level machine connectivity to high-level data analysis and decisionmaking for supervision of industrial processes. Generally, the key enabling technologies can be categorized into connectivity and communication level (CCL), model and semantic level (MSL), and data and knowledge level (DKL), which are as depicted with Fig. 1. The techniques at CCL level, including machine topology and connectivity, M2M messaging protocols, build the data link between machines and allow ubiquitous M2M messaging towards arbitrary interactions. The techniques at MSL level, including ontological modeling and interoperable

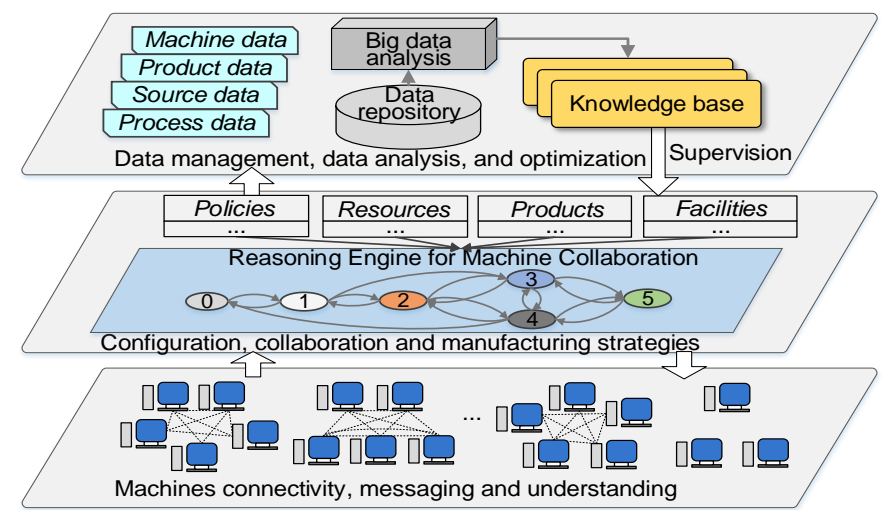

Fig. 1. Key Enabling Technologies of Industrial IoT Applications data presentation and parsing, build the semantic connection and enable inter-machine understanding and collaborations. Then, the techniques at DKL level, including database, knowledge presentation and data analytics, are responsible for data management, analysis, and knowledge-based optimization.

Evidently, the techniques at higher levels are tightly coupled with the underlying supporting techniques at lower levels. Therefore, techniques and methods to gain cross-technical level interoperability have become the major technical concerns.

\section{B. Open Technical Issues}

The enabling technologies have outlined the potential prospects of the manufacturing industry. However, there are technical challenges that keep the heterogeneous technologies from seamless integration. Since IIoT scenarios differ from IoT in many aspects and most technical solutions are for IoT, some underlying challenges exist to hinder the interoperability between machines and the flexibility of industrial applications.

\section{1) Specificities of IIoT}

The manufacturing applications can be considered as a data and communication intensive and highly interactive scenario of IoT applications. The performance of machines parts, connectivity, communication, and data throughput are expected to be more flexible or powerful in manufacturing industry compared to other IoT scenarios, such as smart home and health monitoring, which are summarized in TABLE II. The specificities of IIoT manufacturing industrial networks are:

- Powerful machines with reliable connectivity

- Real-time communication for industrial operations

- Heterogeneous machine network topology

- Machines are tightly intertwined for intensive interactions

- Arbitrary Peer-to-Peer(P2P) or multicast for ubiquitous M2M messaging 
TABLE II

IOT AND IIOT - A COMPARISON OF THE KEY PARAMETERS

\begin{tabular}{|c|c|c|}
\hline Variables & IoT Systems & IIoT Systems \\
\hline $\begin{array}{l}\text { Machine part } \\
\text { platforms }\end{array}$ & $\begin{array}{l}\text { Light-weight embedded } \\
\text { electronics }\end{array}$ & $\begin{array}{l}\text { Light-weight devices/ } \\
\text { standard PCs/IPCs }\end{array}$ \\
\hline Computation power & Light-weight & Light-weight/powerful \\
\hline Power consumption & Normally low power & Depends \\
\hline Network connection & Normally wireless & Wired/wireless industrial \\
\hline Network structure & Normally centralized & Centralized/decentralized \\
\hline Machine interactions & Normally non-intensive & Interaction intensive \\
\hline Messaging type & Client-server/P2P & Arbitrary $\mathrm{P} 2 \mathrm{P} /$ multicast \\
\hline Data throughput & Light-weight & High and varying sized \\
\hline Type of automation & Data fusion based & Machine collaborations \\
\hline
\end{tabular}

- High data throughput with varying sized messages

To achieve arbitrary M2M interactions between the tightly intertwined machines for real-time industrial operations has been a common challenge for heterogeneous machine parts, and therefore become the major technical issues for IIoT systems.

2) Remaining Challenges

The heterogeneity in machine part platforms, flexibility in connectivity, highly interactive messaging between tightly intertwined machines, and high throughput of communication have raised challenges to machine collaborations and manufacturing flexibility. To achieve high-level production flexibility, connectivity and communication level interoperability is a key objective. The underlying technical issues for machine interoperability are:

- Flexible connectivity method for convenient connection, identification, presence and discovery of machines

- Ubiquitous M2M messaging based data and event interactions for collaborative automation of machines

- Data presentation and inter-machine understanding for interoperable manufacturing operations

- Modeling for high-level manufacturing management

However, with respect to flexible manufacturing, higher level functions regarding the system configuration, overall resource modeling, production strategy, product data analysis, and domain-knowledge based optimization are critical building blocks. These high-level functionalities are enabled by intermachine communication and understanding.

\section{Industrial Standards and Related Work}

The integration of IoT technologies with industrial networks has attracted much research interests in both academia and industry. The lack of architecture standards for industrial Internet and connectivity in the IoT is considered a major threat [5]. Therefore, many Standard Development Organizations (SDOs) are producing industrial standards and frameworks for industrial applications, such as IEEE, ETSI, IETF, 3GPP, oneM2M, Microsoft manufacturing group [6]. These industrial standards mainly focus on the M2M communication protocols and high-level manufacturing management.

\section{1) Technological Convergence in the Industry}

It is significant to bridge the gap between Operation Technology (OT) and Information Technology (IT) to support inter-machine collaborations and enhance production efficiency and flexibility. Different perspectives of IT and OT professionals are a typical reason for making the integration a challenge [7]. This is due to the heterogeneity of machine platforms and insufficient inter-machine communication and understanding. Networking of OT is determined by the functions and inter-relationships of machines, and IT networking is the building blocks for flexible OT functions. Industrial communication is regarded as the backbone of the IT/OT integration, and pioneers, such as SIEMENS and Atos Worldgrid, are making encouraging progresses.

The machine connectivity and messaging techniques are the foundations for the collaborative automation between machines and the flexibility of manufacturing operations. However, investigations of the contemporary manufacturing have gone beyond machine collaborations, and collaborative robots (CoBots) are now being considered as practical industrial solutions, which allow human-to-robot interaction in the same workspace. Robot kinematics and manufacturing tasks

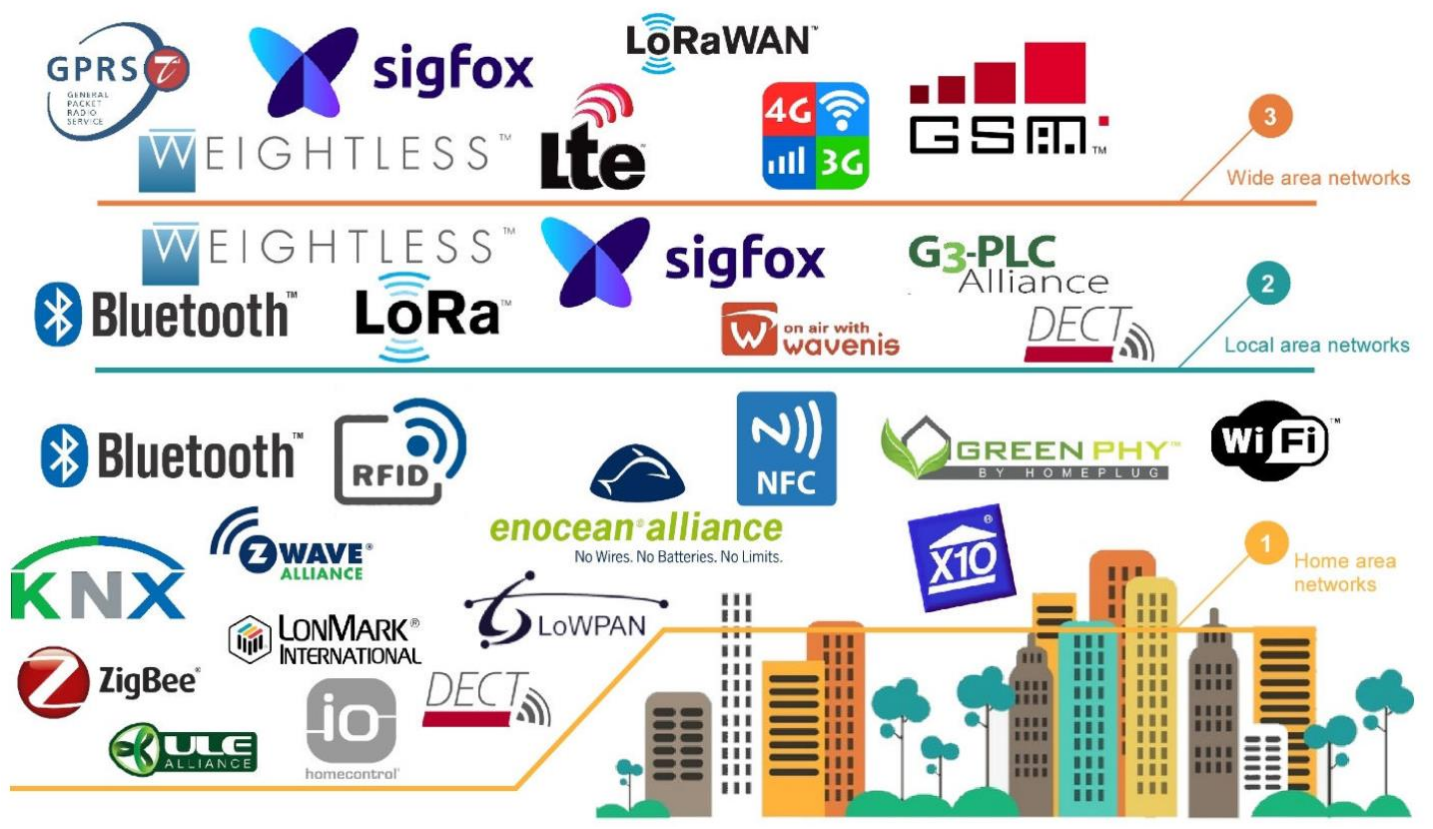

Fig. 2. Wireless Communication Solutions for Different IoT Applications Scenarios [11] 
optimization are typical research topics. For instance, a novel unified reconfigurable kinematic model is proposed in [8] for Left and Right collaborative robotic arms to reduce the number of mathematical steps involved in forward kinematics, which increases the efficiency by reducing the time consumed. A flexible ontology-based task decomposition strategy is introduced in [9] for characterizing and assessing the task-based safety of human-robot collaborative manufacturing tasks. This investigation focuses on the underlying techniques for intermachine collaborations to pursue manufacturing flexibility.

\section{2) Standards, Middleware and Application Programming} Interfaces (APIs)

Some communication interfaces and service platforms are being standardized for industrial applications by SDOs. Technical solutions and standards have been invented for application specific contexts, such as radio communication standards WiFi, 3G/4G/5G, LTE, and network protocols WirelessHART, ISA100.11a, and Z-Wave [10]. The wireless $\underline{\text { communication solutions for different IoT application scenarios }}$ are summarized in Fig. 2 [11]. A number of messaging technologies have been invented to support the connection of devices for industrial applications in a distributed network, including Direct Distribution Service (DDS), Message Queuing Telemetry Transport (MQTT), Advanced Message Queuing Protocol (AMQP), Java Message Service (JMS), Representational State Transfer (REST), Constrained Application Protocol (CoAP), Extensible Messaging and Presence Protocol (XMPP), Zero MQ (ZMQ), MTConnect, and OPC UA [12-14]. The OPC Foundation is revolutionizing its standard of communication between industrial equipment with the latest OPC UA specifications, which can be deployed on all types of platforms [15]. The technologies have their specific strengths in dealing with different situations, such as lightweight messages, low-power devices, real-time performance, and service discovery. The method for choosing the optimal technique should be based on the understanding of both the system architecture and requirements of specific applications.

Manufacturing Reference Architectures (MRAs) regarding open M2M system framework, manufacturing service architecture, and business models are also critical building blocks. ETSI M2M service architecture is the first step towards a universal M2M platform, which has already provided a good maturity level on unified communication capabilities and protocols [16]. Smart Manufacturing Leadership Coalition (SMLC) is building an open smart manufacturing platform for collaborative industrial networked information applications [17]. OneM2M global initiative is established to produce M2M service layer specifications to ensure M2M devices can communicate on a global scale [18]. In addition, Microsoft manufacturing group inaugurated a cloud-based framework that connects smart devices across manufacturing networks emphasizing user interfaces, enterprise-class social-computing and security-enhanced solutions, named Reference Architecture for Discrete Manufacturers (DiRA) [19].

Although they might stress different properties, they share several common concerns including integration of $\mathrm{M} 2 \mathrm{M}$ techniques, highly interconnected machines, open framework, manufacturing intelligence, etc.

\section{3) Related Work}

The M2M technologies have gained much research interest in various IoT applications. In particular, massive random access management is considered one of the most critical challenge. By comparing the key parameters of the state-of-theart solutions, a collision resolution access model for massive Machine Type Communication (MTC) over LTE/LTE-A is proposed in [20] to resolve the preamble collisions. Then, hybrid M2M random access protocols combining collision avoidance and tree collision resolution random access is introduced in [21] to handle synchronized M2M traffic in LTE wireless systems. In addition, application level modeling of ubiquitous M2M that fits the tightly intertwined machine networks scenario has been a technical issue. A semantic data model and resource-based ubiquitous data accessing method is designed to acquire and process IoT data ubiquitously [22]. In order to serve dynamic applications across different platforms, a naming, addressing, and profile server (NAPS) as a middleware is proposed to bridge different platforms in IoT sensory environments [23]. In addition, a M2M messaging mechanism for ubiquitous data access and event notification of IIoT applications is presented with experimental studies in [24], which shows certain flexibility in machine presence, discovery and ubiquitous M2M messaging.

With respect to MRA, appropriate designs based on RESTful, SOA, and Cloud service are introduced to tackle the complexity, dynamics and uncertainties of IIoT manufacturing. In order to support different applications, a configurable information service platform is proposed for the development of IoT-based applications by combining ontology and RESTful service [25]. Based on a comparison and analysis of existing M2M platforms, an M2M service platform architecture (M2SP) is proposed in [26] consisting of a Device-, User-, Application- and Accessplatform, where entities interact with each other and provide M2M service platform functionalities. To enable rapid rescheduling and reconfiguration of production systems, ontology based Adaptive Production Management (ARMU) is proposed for adaptability and on-the-fly responsiveness in production management by combining multi-agent and network-centric software [27]. A Smart Manufacturing Network (SMN) reference architecture and platform is developed for highly connected, knowledge-enabled manufacturing toward production flexibility [28]. A Cloud Computing and IoT-based Cloud Manufacturing (CCIoT-CMfg) service system is proposed for sharing, circulation, use and allocation of resources and capabilities[29].

In addition, the modeling of products, manufacturing process, and production context, and knowledge representation mechanism are also crucial issues for manufacturing flexibility. Ontology has gained widely acceptance to establish the relationship between concepts for computation in particular domain of discourse, which is considered as a promising tool to organize information and limit complexity. Some proactive practices are conducted by researchers and practitioners and encouraging results are obtained in related investigations. Knowledge management (KM) is integrated to quality management (QM) in [30] to improve a firm's performance for large manufacturing organizations, where system engineering aspects such as operational efficiency improvement and system 
performance are leveraged in the enhanced QM system. The effectiveness of such a KM/QM strategy is demonstrated through quantitative empirical research. In order to promote the semantic interoperability of oneM2M architecture, an expressive ontology IoT-O is proposed in [31] to make use of already defined ontologies by adding some missing concepts. In order to meet production requirements for fast iteration and realize agile and efficient manufacturing resource allocation, an ontology-based resource reconfiguration method is proposed from the perspective of resource utilization in [32]. Addressing the communication and comprehension challenges in Industry 4.0, the concept of Semantic I4.0 component is proposed to develop a digital presentation of information about an object with semantic knowledge presentation formalisms [33]. The ontological models of manufacturing domain can potentially promote the understanding of resources and capabilities, and therefore introduce flexibility and intelligence.

Although the technical issues have been sufficiently addressed, there still exist underlying gaps between information systems and physical machine operations. The lack of wellsuited production line scale strategies to enhance machine interoperability is identified as a major challenge to achieve higher level flexibility. Flexible connectivity, ubiquitous messaging, and cross-platform understanding are the central issues to manufacturing flexibility. However, the requirements in flexible connectivity, presence and discovery, ubiquitous M2M messaging and understanding of the highly intertwined and communication intensive machines with heterogeneous platforms have raised new challenges.

\section{The Scope of this Investigation}

In view of IoT solutions for manufacturing industry from system and software perspective, this investigation endeavors to provide customized solutions to gain machine interoperability and manufacturing flexibility through production line-level inter-machine collaborations focusing on: (1) modelling and theoretical analysis of inter-machine collaborative automations in industrial networks; (2) machine modularization and topology structure for communication intensive applications; (3) ubiquitous M2M messaging and inter-machine understanding techniques for collaborative automations; (4) model-based management for applicationlevel adaptation and manufacturing flexibility.

To maximize manufacturing flexibility, the custom designs for the specificities of manufacturing applications to gain machine interoperability are the central technical issues.

\section{Modeling AND TeChNicAl SOlutions}

In order to realize the envisaged flexibility based on machine collaborations, this section models the communication intensive collaborative automations of industrial networks and proposes some custom designed technical solutions. Regarding the industrial applications as interactive information systems, a functional model is proposed to describe the data interaction mechanism and inter-relationship between machines. Then, the technical solutions contributing machine interoperability and manufacturing flexibility are illustrated.

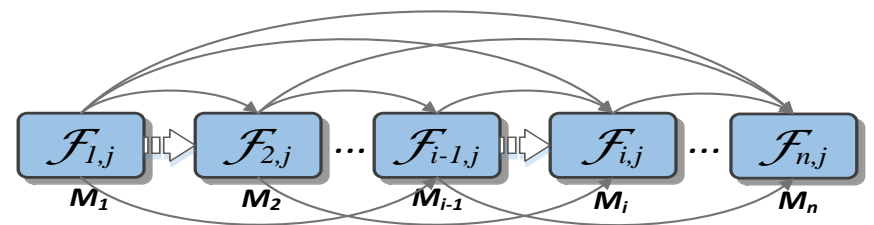

Fig. 3. Machine Collaboration Model of IIoT Systems

\section{A. The Functional Model of Machine Collaborations}

In view of the technical solutions for manufacturing systems, it is necessary to describe the components and their interrelationship, namely the roles of machines, how they generate, exchange, and consume data to fulfil the interactive operations. From a system perspective, industrial applications can be regarded as information systems where each machine is a processing unit which takes previous machines' output data or events as its inputs and creates new variables as the outputs.

As shown in Fig. 3., suppose there are $n$ functional modules or machines in an industrial application denoted by $M_{1}, M_{2}, \ldots$, $M_{n}$, and the functions of $M_{i}(i \in(1, n))$ are denoted by $\mathcal{F}_{1, j}, \mathcal{F}_{2, j}, \ldots$ $\mathcal{F}_{n, j}$, where $j$ is the batch number of production. Define $C_{i, j}$ the initial configuration of $M_{i}$, the parameters of $k^{\text {th }}$ final product $\mathcal{P}_{(}(k)$ processed by $M_{l}$ to $M_{n}$ can be presented with:

$\mathcal{P}_{j}(k)=\sum_{i=1}^{n} \mathcal{F}_{i, j}\left(C_{i, j}, I_{i, j}(k)\right)$

where $F_{i, j}\left(C_{i, j}, I_{i, j}(k)\right)$ means the industrial process of $k^{\text {th }}$ product in $j^{\text {th }}$ batch on $i^{\text {th }}$ machine under configuration $C_{i, j}$ and input $I_{i, j}(k), I_{i, j}(k)$ means the input of machine $M_{i}$, and,

$I_{i, j}(k)=\left(F_{1, j}(k), F_{2, j}(k), \ldots, F_{i-1, j}(k)\right)^{T} \times A_{i, j}$

In (2), $F_{i, j}(k)=\mathcal{F}_{i, j}\left(C_{i, j}, I_{i, j}(k)\right)$ and the coefficient vector $A_{i, j}$ $(i \in(1, n))$, defined in the application specific configurations, clarifies the relationship between $M_{i}$ and $M_{l}$ to $M_{i-1}$. For example, if $A_{i, j}=(1,0, \ldots, 0,1)$, then $I_{i, j}(k)=\left(F_{1, j}(k), \boldsymbol{0}, \ldots, \boldsymbol{0}, F_{i-}\right.$ ${ }_{1, j}(k)$ ), which means machine $M_{i}$ only needs output parameters of $M_{l}$ and $M_{i-l}$ as its input.

Combining (1) and (2) can obtain the following equation:

$\mathcal{P}_{j}(k)=\sum_{i=1}^{n} \mathcal{F}_{i, j}\left(C_{i, j},\left(F_{1, j}^{k}, F_{2, j}^{k}, \ldots, F_{i-1, j}^{k}\right)^{T} \times A_{i, j}\right)$

Evidently, the industrial processes of the machines are accomplished sequentially and some machines use previous machines' operation results as inputs. Therefore, collecting results of previous machines in real-time with a ubiquitous messaging mechanism is a central issue to the success of collaborative industrial applications. By appropriately modeling the machine relationships, machine interactions, machine configurations, manufacturing policies, and defined manufacturing operations can be collaboratively fulfilled with the interactive machines.

The online generated data can be collected and saved in a data repository, and the analytical insights can potentially add intelligent optimizations to the system. The matrix of machine generated data can be presented with the following equation: 


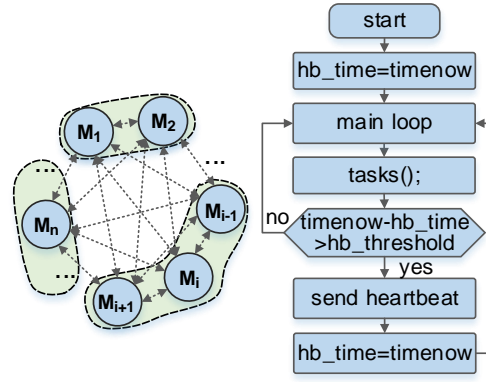

(a)

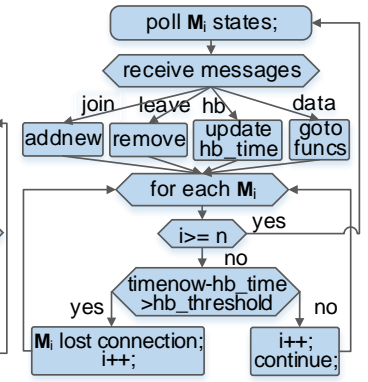

(c)
Fig. 4. Heartbeat-based Machine Presence and Discovery. Mi denotes the machine items, and $\mathrm{hb}$, hb_time, and hb_threhsold represent heartbeat, heartbeat time, and heartbeat interval, respectively.

$$
\left(\mathcal{F}_{i}, C_{i}, A_{i}\right) \leftarrow\left[\begin{array}{cccc}
\mathcal{F}_{i, 1} & C_{i, 1} & A_{i, 1} & \left(\mathcal{P}_{1}(1), \mathcal{P}_{1}(2), \ldots, \mathcal{P}_{1}(m)\right) \\
\mathcal{F}_{i, 2} & C_{i, 2} & A_{i, 2} & \left(\mathcal{P}_{2}(1), \mathcal{P}_{2}(2), \ldots, \mathcal{P}_{2}(m)\right) \\
\ldots & \ldots & \ldots & \ldots \\
\mathcal{F}_{i, \ell} & C_{i, l} & A_{i, l} & \left(\mathcal{P}_{\digamma}(1), \mathcal{P}_{\zeta}(2), \ldots, \mathcal{P}_{\digamma}(m)\right)
\end{array}\right]
$$

where $l$ and $m$ are the quantity of batches and the number of products in a batch. The machine functionalities, machine configurations, input and output relationship, and product parameters can be used for further analysis. The functionality of machines, its configuration, and the correlation matrix could be refined to produce optimal performances by taking advantage of collected data with data analysis methods.

This functional model gives a clear description of the collaborative automations of tightly intertwined machines in communication intensive manufacturing industrial networks. It provides guidelines to gain machine interoperability and manufacturing flexibility with the underlying messaging, understanding and modeling technologies. Practical industrial systems may conform to more complex models where machines are in both parallel and serial forms.

\section{B. Technical Solutions and Design Principles}

In pursuance of efficient data interactions and dynamic collaborations between machines, the design principles and technical solutions that are well-suited for the above presented functionalities are illustrated.

\section{1) Decentralized Structure and Machine Modularization}

Compared to the other IoT applications, the manufacturing systems are more communication intensive, where interactive collaborations can effectively maximize production efficiency. Traditional infrastructure-based or Ad hoc connectivity and centralized service architecture are not suitable for the highly intertwined machines. Therefore, a decentralized connectivity is designed to eliminate the limitation of central controllers and allow ubiquitous M2M messaging between machines.

The decentralized machines play different roles, either observe variables or control motions, either generate data or consume data, either work loosely or tightly coupled with peer machines. A group of machines might work closely on one particular task with their collaborative operations. The roles of the machines and their inter-relationship need to be defined for efficient collaborations in between. Therefore, the machines are categorized into three roles: device, module controller, and coordinator.
- Devices are individual machines working on particular manufacturing tasks or part of tasks of a module.

- Module controllers are controller devices in groups of tightly coupled machines working on particular tasks.

- Coordinators are higher level machines or modules responsible for the operation of the overall system.

The three roles can reshape the machines of an industrial system into a hierarchical structure, where lower-layer devices, medium-layer modules, and higher-layer coordinators can efficiently interact with each other and collaboratively perform the operations. With this design concept, an industrial system consists of functional modules, either individual machines or groups of devices, and overall coordinators.

The decentralized structure introduces more freedom to machine interactions and therefore could maximize the efficiency of machine collaborations. The modularization of machines simplifies the machine interaction model by regarding module as a functional element and separate the machine interactions into inter-module and intra-module interactions and inter-device interactions.

\section{2) Machine Presence and Discovery}

For efficient data interaction, each machine needs to keep the peer machines updated of its state and be aware of the states of the peers. The machine presence and discovery are critical issues for efficient messaging between machines. Machine identification and heartbeat-based machine presence and discovery methods are employed to manage the availability and awareness of the connected machines in an industrial network.

The heartbeat mechanism is as illustrated in Fig. 4. As shown in Fig. 4(a), each machine can send heartbeats to the peers to notify its state and receive heartbeats from all peers to observe their states. The machines send heartbeats with the logic presented in Fig. 4(b). Heartbeats are sent according to the system time of the machines. If the time interval between the current time timenow and the last heartbeat $h b \_t i m e$ surpasses the defined heartbeat interval $h b \_t h r e s h o l d$, a heartbeat is sent and $h b \_t i m e$ is set to timenow. The received heartbeats handling method is as shown in Fig. 4(c). All machines are distinguished with Universal Unique Identifiers (UUIDs), and each machine builds a buffer to observe the real-time updates of peer machines' states. The messaging events join, leave, heartbeat, and data denote a machine joining in the network, leaving the network, sending a heartbeat, and sending data. When a new machine joins in a machine network, all the peers can receive its join message. The new machine can also receive messages of the existing machines to observe their states. With heartbeat event, the machines can determine the connectivity states of the peer machines. When a machine leaves a network, all peers remove the relevant information in their observation buffers.

With this heartbeat mechanism, machines can observe the peers' states and keep all the peers updated of their states.

\section{3) Ubiquitous M2M Messaging Mechanism}

The real-time $\mathrm{M} 2 \mathrm{M}$ messaging is a prerequisite of data interaction based machine collaborations and further flexibility. In order to enhance the interactive collaboration of machines, the ubiquity of M2M messaging is a critical parameter. 


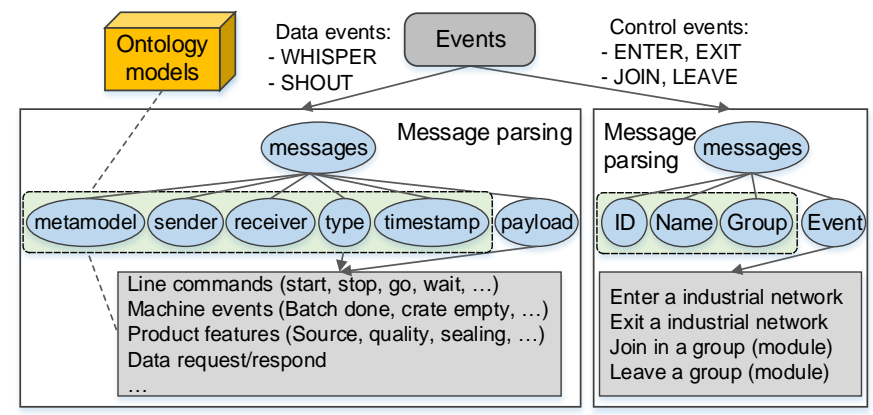

Fig. 5. Data Presentation for Inter-machine Understanding

Therefore, $\mathrm{P} 2 \mathrm{P}$ and multicast messaging functions are introduced to achieve arbitrary $\mathrm{M} 2 \mathrm{M}$ messaging:

- whisper (UUID, event, message)

- shout (groupID, event, message)

whisper and shout are used for P2P and multicast communications, respectively. Parameters UUID and groupID are the object machine or group of machines as a module, event is the pre-defined type of messages such as enter, exit, join, leave, request, reply, and notify, and message is the payload of data to send. With this arbitrary M2M messaging method, each machine can initiate a P2P message to any connected machines, it can also initiate a multicast to any permitted modules.

The P2P and multicast based request, reply, and notification potentially allows the flexible ubiquitous communication between the machines in an industrial network for collaborative automations. This P2P and multicast messaging enables the ubiquitous interactions between the decentralized machines.

\section{4) Data Presentation and Inter-Machine Understanding}

The appropriate modeling and presentation of data could enhance the interoperability between machines which are critical for interactive operations. Due to the light-weightness, arbitrary structure data presentation, and widely acceptance by different software platforms, Javascript Object Notation (JSON) is used to structurize product data and present the ontological models toward mutual understanding of machines.

The message structure is designed as shown in Fig. 5. A header metadata describes some essential information for efficient communication with a fixed structure, and the effective data is enclosed in the payload node that is flexible in structure. With the metadata description of the messages, machines can identify where the message is from, where it goes, what type the message is, etc. The machines can parse the JSON message in a correct way and do further steps by knowing the metadata first. Then, provided the type of message is known, the machine can understand the format of payload node and parse the variables. Therefore, this metadata based message structure can effectively enhance the understanding between machines by normalizing the data with pre-defined formats.

\section{Model-based Design for Flexible Industrial Applications}

The manufacturing flexibility lies in the reconfigurability of system supported by machine interoperability, including the reconstruction of system structure, restructuring of production process, and reconfiguration of machine functionalities. Based on the ubiquitous communication and interoperable understanding of machines, the flexibility can be explored in

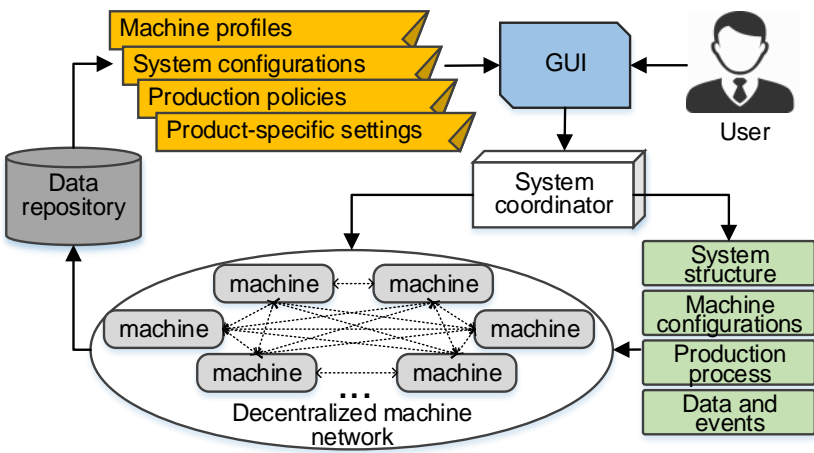

Fig. 6. Design Model for Flexible Industrial Applications

higher level functionalities. Fig. 6 depicts the model-based design of a reconfigurable system in a decentralized machine structure. The key design principles are summarized as follows:

- Modularized system design and open system framework

- Identifiable machines and ubiquitous M2M messaging

- Global modeling of machines and industrial processes

- Reconfigurable machine functionalities

- Efficient Graphical User Interface (GUI) for system reconfiguration

It is evident that the modularized design can divide the whole system into inter-related functional modules, and Finite State Machine (FSM) can be used to model the interactions and interrelationships between machines. The identifiable and state observable machines with open communication interfaces can realize the interactions between modules and their collaborative automation. A global modeling of the overall system details the manageable parameters of the modules that can be flexibly changed with users. An efficient GUI provides users with an interface for high-level management of overall system.

In this section, the functional model describes the interrelationships of machines in manufacturing industrial networks, the corresponding solutions promise machine interoperability for communication intensive machine systems, and the design principles outline the blueprint for manufacturing flexibility.

\section{DESIGN AND IMPLEMENTATION IN PICKNPACK}

The presented concept and solutions are implemented to gain production line scale manufacturing flexibility in the PickNPack food manufacturing line. The EU PickNPack project aims to integrate the state-of-the-art smart devices with

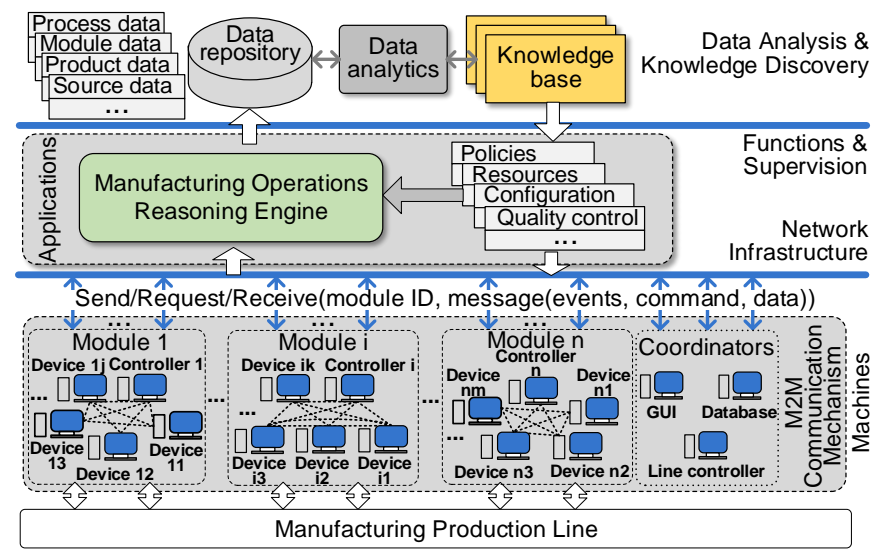

Fig. 7. Architecture for Collaborative Machine Networks 


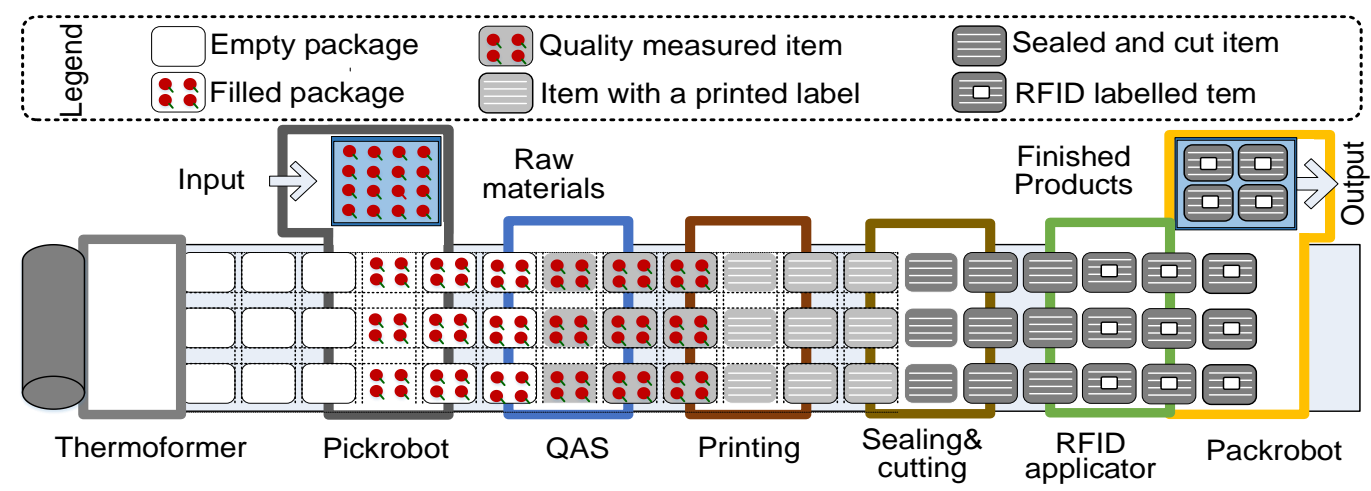

Fig. 8. Schematic Diagram of PickNPack Food Manufacturing Line

the emerging ICTs toward a flexible digital food manufacturing production line. This section illustrates the design and implantation of PickNPack line with the presented solutions.

\section{A. System Architecture for Collaborative Automations}

With the modular design principle, the system architecture for PickNPack is as shown in Fig. 7. The machine system is modularized according to the manufacturing functionalities in a decentralized structure. The devices work in a collaborative way to constitute functional modules to perform particular manufacturing operations. The coordinators such as GUI, line controller, and database are responsible for the management of the overall system. Machine configuration and reasoning logic can be configured to the machines with coordinators. Production data is collected and stored to data repository, and analytical insights can be applied to supervise the manufacturing.

In line with this reference architecture, the PickNPack line as shown in Fig. 8 is divided into eight functional modules:

1) Thermoformer(M1) for online food package fabrication

2) Pickrobot(M2) to pick up raw food and place it in packages

3) Quality assessment \& sensing(QAS)(M3) for product quality evaluation

4) Printing(M4) for dynamic label printing

5) Sealing\&cutting(M5) to seal and cut the labels

6) Packrobot(M6) to pick and place products in output crates

7) Information traceability (M7) for data tracking and tracing

8) Cleaning(M8) to clean the line with flushing water

These modules each fulfill their specific functions with one or multiple machines. For example, QAS module includes five sensors (RGB, 3D, hyperspectral, microwave, and X-ray) for food quality evaluation. The QAS module controller collaborates with the sensor devices for data fusion and decision making. The food manufacturing processes from package fabrication to outgoing batches are accomplished by the functional modules and their collaborative automations.

\section{B. Product Identification and Operation Synchronization}

For the production operations, machines need to know the IDs of products in its workspace and integrate produced data. In this project, a mechanical encoder is used to measure the position of packages to multicast to all machines in the line. The machines identify the products in their workspaces by calculating the encoder position. A Radio Frequency Identification (RFID) system is applied to identify input/output crates to integrate the raw material and outgoing information.
By assigning a specific UUID to each product unit, data produced in the line can be integrated. Therefore, machines can request data from peer machines with product UUIDs. The arbitrary ubiquitous data access and product identification allows the collaborative automations between machines to efficiently conduct the production operations.

For the synchronization of machine operations, each machine has a built-in Finite State Machine (FSM) to control the operation states, which includes three states: "Idle", "Ready" and "Go". The machines turn to "Go" state and send out a "Go" message to the peer machines when they receive new messages and start new manufacturing operations, and turns to "Ready" state and send out "Ready" message when they finish the manufacturing operations. When all machines are ready for the next operation, Thermoformer (M1) can start a new run.

\section{Ubiquitous Messaging in PickNPack}

For machine presence, discovery and ubiquitous messaging, an open source framework for P2P communication called Zyre is chosen for machine messaging in the PickNPack line. Zyre handles group messaging over local area networks with no administration or configuration, where the peers talk to each other without central brokers or servers. This makes it very suitable for controlling distributed machines in a decentralized structure. Machines can initiate periodic heartbeats to notify the peers for presence and receive heartbeats to discover the peers. By being aware of the peers, they can initiate $\mathrm{P} 2 \mathrm{P}$ and multicast messages for data interactions and event notifications.

Distinguished with UUIDs, machines can request, reply, receive, and notify the peers concerned in the execution of their tasks. With the proposed data presentation method, machines
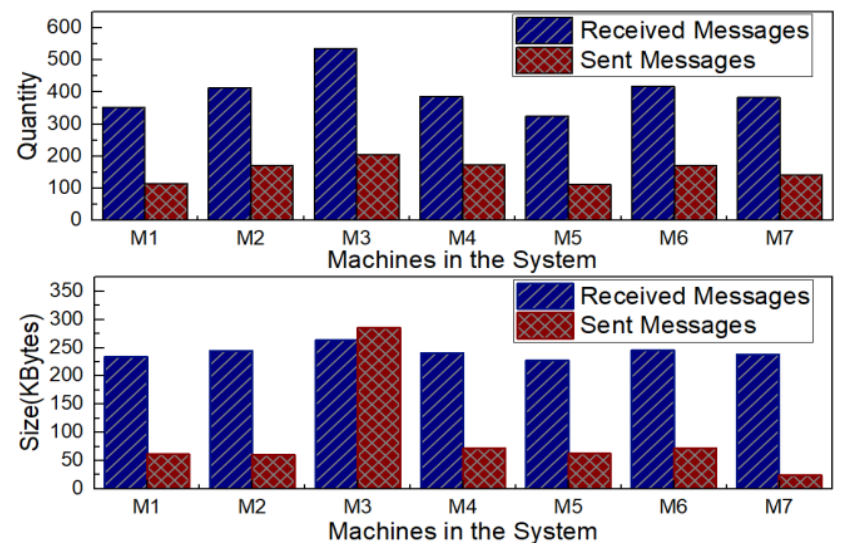

Fig. 9. Quantity of M2M Messages and the Total Size of Messages 


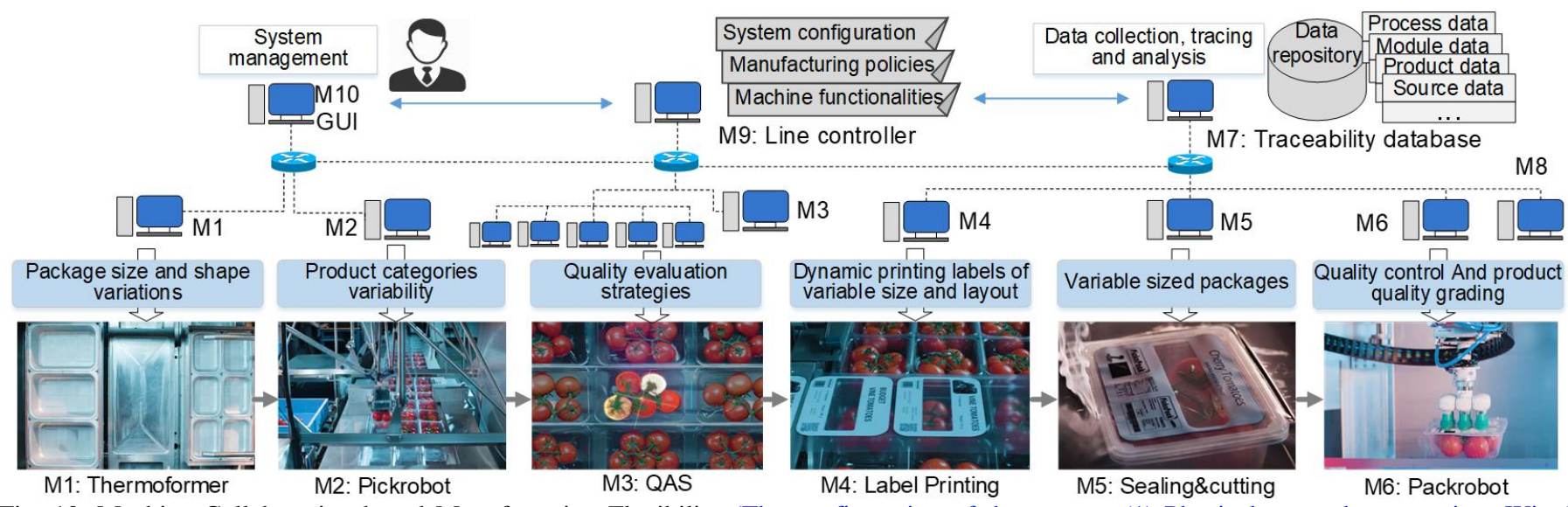

Fig. 10. Machine Collaboration based Manufacturing Flexibility (The configuration of the system: (1) Physical network connection: Wired Internet or WiFi; (2) Messaging protocol: Zyre based on ZMQ, via P2P and multicast; (3) Controller machines: PC, PLC, or PC and PLC; (4) PC operating systems: Windows or Ubuntu. (5) Software environments: Visual C\#, C++, or LabVIEW.)

wrap the data to send to the peers and the receivers can easily parse the messages and obtain the variables. Fig. 9 gives the quantity and total size of received and sent messages of modules M1 to M7 in PickNPack line in 1 minute. Since the quantity of received heartbeat messages of a machine is much more than it sent out, the received messages' size and quantity are much bigger than the sent ones. However, for M3 which includes five sensor devices, the size of configuration file it sends out are much larger than the heartbeat messages. Therefore, the quantity of received messages of M3 is larger than the sent ones, while the size is smaller.

\section{System Performance and Flexibility of PickNPack Line}

By implementing the proposed technical solutions in system architecture design, through-the-line product identification, synchronization of industrial operations, and ubiquitous M2M messaging, a flexible PickNPack food manufacturing line is successfully achieved. The achieved system was demonstrated with fresh and processed food, tomatoes and chicken breasts. The speed was 17 packages per minute, and the bottleneck was the Pickrobot which picked and placed per food item every 3.53 Seconds. The other modules were able to speed up to the set goal of 30 packages per minute by completing the information processing and manufacturing tasks in 2.0 seconds, which is considered state-of-the-art. The single M2M communication and message wrapping/parsing can be completed in milliseconds or tens of milliseconds. The system can speed up

TABLE III

Manufacturing FleXibiLities OF PickNPack Line

\begin{tabular}{|c|c|}
\hline Added Flexibilities & Descriptions \\
\hline $\begin{array}{l}\text { System structure and } \\
\text { manufacturing policy }\end{array}$ & $\begin{array}{l}\text { The system structure and production process can be } \\
\text { reconstructed }\end{array}$ \\
\hline $\begin{array}{l}\text { Multiple product } \\
\text { categories }\end{array}$ & $\begin{array}{l}\text { Multiple food categories are supported with fast } \\
\text { change over, including vegetables, fruits and ready } \\
\text { meals }\end{array}$ \\
\hline $\begin{array}{l}\text { Reconfigurable } \\
\text { Machine functionality }\end{array}$ & The functionalities of machines are reconfigurable \\
\hline $\begin{array}{l}\text { Variable package size } \\
\text { and shape }\end{array}$ & $\begin{array}{l}\text { Modular element mold allows variable sizes and } \\
\text { shapes of food packages }\end{array}$ \\
\hline Dynamic label printing & $\begin{array}{l}\text { The layout and information of printed labels are } \\
\text { variable and selectable }\end{array}$ \\
\hline $\begin{array}{l}\text { Adaptive quality } \\
\text { evaluation strategy }\end{array}$ & $\begin{array}{l}\text { Multiple quality measurement and assessment } \\
\text { schemes for different product types }\end{array}$ \\
\hline $\begin{array}{l}\text { Adjustable quality } \\
\text { control and grading }\end{array}$ & $\begin{array}{l}\text { Quality control settings for product grading and } \\
\text { classification are adjustable }\end{array}$ \\
\hline
\end{tabular}

to reach the goal by increasing the capacity (motion planning) of the robot to reach 2.0 seconds per pick-and-place task. The change-over and reconfiguration time for the whole line was 5 minutes [34]. Compared to existing manufacturing automation network systems, the performance of the system has been largely improved and some added flexibilities are obtained.

\section{1) The System Performance of PickNPack Line}

In the first place, the automation level of the manufacturing has been largely improved. Normally, the manufacturing functions may need about six to ten operators. With this reconfigurable system, it minimally needs two operators: one at the raw material feed-in workspace of PickRobot, and the other is at the output workspace of PackRobot. The ubiquitous M2M interactions allow collaborative automations of functional machines under an initial configuration and production strategy, which has eliminated people at the workspaces of the machines. Due to the high performance of functional machines and realtime ubiquitous M2M messaging protocols, the reliability of the system satisfies the developers after repeated long-running tests.

In addition, some added merits that is not possible with traditional techniques are obtained. The ubiquitous machine interactions and open system architecture allow users to configure and reconfigure the system conveniently by updating the configuration with GUI. The system can be reconfigured to accommodate different product types and manufacturing policies. The machines access configuration models in the system initiation stage of production jobs. The flexibility of the system can potentially introduce minimum human interventions, high quality automation, and easier production line-level system maintenance. The system performs satisfactory Overall Equipment Effectiveness (OEE) in systematic tests of each manufacturing task, including packaging fabrication, raw material picking, quality sensing, label printing, sealing and cutting, output packing, and information tracking and tracing.

\section{2) The Added Flexibilities of PickNPack Line}

The collaborative manufacturing operations between the modules and machines in a module have introduced highly adaptive automations and potential flexibility to the PickNPack line. Fig. 10 describes the production flexibility leveraged by machine modularization, decentralized structure, ubiquitous messaging, flexible data presentation, and model-based management. The added flexibilities of PickNPack line 
obtained by the proposed techniques are given in TABLE III. In summary, (1) the system structure and manufacturing policy can be reconstructed; (2) multiple product categories are supported with a fast change over; (3) the functionalities of machines are reconfigurable for different product categories; (4) the size and shape of food packages are adjustable; (5) the layout and information of label printing are adjustable; (6) quality sensing and quality assessment strategies are adaptive; and (7) the product quality control and grading methods are adjustable.

The PickNPack food packaging line has been successfully demonstrated to the public in Wageningen UR, Wageningen, Netherlands, and National Center for Food Manufacturing, Holbeach, UK.

\section{DISCUSSIONS}

This investigation highlights the features of machine systems in manufacturing industrial networks, and pursues production line scale flexibility focusing on machine modularization and connectivity, presence and discovery, ubiquitous messaging and understanding, and model-based management. The modular design and decentralized structure extends the degree of freedom for machine interactions, and eases maintenance and upgrade of the whole system. The ubiquitous data interactions allow collaborative automation and optimization. The interoperable data interaction introduces flexibilities to the industrial applications in high-level system management. Therefore, some promising information theories and techniques, such as machine learning and big data analysis, are made possible to be applied for pursuing intelligent optimizations.

The design of this work is system and software centric which aims to bridge OT and IT with communication and semantic technologies and gain system flexibility. The solution is based on the premise that machines are computerized with certain computation power and open communication interfaces. Another issue is the lack of common modeling formalism for the interoperable understanding between machines. PickNPack defines machine profiles, message formats, and machine configurations, manufacturing process, etc. with a global description model named 'worldmodel'. A further issue in terms of flexible manufacturing is the appropriate use of domain knowledge for some product-specific applications. Therefore, product domain knowledge such as suitable package size, robot grippers, sensors, and quality evaluation methods for different food categories are all significant to the highly adaptive manufacturing. In addition, the underlying mechanical level integration may also be a tough job, which is significant to the machine interoperability and manufacturing flexibility.

Although many technical proposals are found, we can hardly find practical solutions integrating M2M technique with system modeling and architecture design for machine interoperability and system flexibility. Most solutions highlight the flexibility in high-level entities and resource management without customized M2M techniques. For instance, M2SP [26] focuses on the management of devices, users, applications and access above the core machine networks; ARMU project [27] focuses on the underlying semantic reasoning to support event handling for high-level resource management; SMN reference architecture and platform [28] allow system scheduling and reconfiguration, but focuses on knowledge-intensive management above the M2M network; CCIoT-CMfg [29] integrates IoT and Cloud systems without specific M2M solutions for machine networking. This investigation provides valuable insights for practicing manufacturing flexibility by constructing a collaborative automation network with systematic technical solutions.

\section{CONCLUSION}

This paper determines the specificities of manufacturing IIoT applications, describes collaborative automation paradigm with a proposed functional model, and provides customized technical solutions for manufacturing flexibility. The presented designs introduce the emerging ICTs and IoT concepts to gain interoperability between the tightly intertwined and communication intensive machines, and the practices in PickNPack line have demonstrated the feasibility.

The presented work can be regarded as a successful attempt to apply emerging technologies and computing paradigms to innovate industrial networks in the way of machine operations. The presented methods can effectively improve production line scale machine interoperability that is fundamental to manufacturing flexibility and potential intelligence.

The lessons learned from the PickNPack practice lie in: (1) mechanical integration and machine computerization are tricky but indispensable for high-level interoperability and flexibility; (2) the presented machine modularization, decentralized structure, ubiquitous $\mathrm{M} 2 \mathrm{M}$ and semantic modeling can effectively enhance efficiency and interoperability of machines to allow flexible manufacturing; and (3) practical applications may need specific domain knowledge of the products for optimal configuration, data analysis and system optimization.

The design concept and technical solutions presented in this work might be of interest for researchers and practitioners to innovate IIoT investigations and applications.

\section{REFERENCES}

[1] Y. Liao, E. F. R. Loure, F. Deschamps, "Industrial Internet of Things: A Systematic Literature Review and Insights," IEEE Internet of Things Journal, vol. 5, no. 6, pp. 4515-4525, 2018.

[2] J. Zhou, P. Li, Y. Zhou, B. Wang, J Zang, L. Meng, "Toward NewGeneration Intelligent Manufacturing," Engineering, vol. 4, pp. 11-20, 2018.

[3] E. Sisinni, A. Saifullah, S. Han. U. Jennehag, M. Gidlund, "Industrial Internet of Things: Challenges, Opportunities, and Directions," IEEE Trans. Ind. Informatics, vol. 14, no. 11, pp. 4727-4734, 2018.

[4] G. Arnold, "Intelligent Systems: A New Industrial Revolution," IEEE Electrif. Mag., vol. 4, no. 1, pp. 63-64, 2016.

[5] M. Weyrich and C. Ebert, "Reference Architectures for the Internet of Things," IEEE Softw., vol. 33, no. 1, pp. 112-116, 2016.

[6] D. Famolari and D. Ventures, "M2M Communications for E-Health and Smart Grid: An Industry and Standard Perspective," IEEE Wirel. Commun., vol. 21, no. 1, pp. 30-37, 2012.

[7] Siemens Industry, Inc., "Connectivity: Backbone of the Digital Enterprise," 2017. [Online]. Available: https://www.industry.usa.siemens.com/ automation/us/en/industrial-communications, Accessed: Jan. 06, 2019.

[8] A. M. Djuric, R. J. Urbanic, J. L. Rickli, "A Framework for Collaborative Robot (CoBot) Integration in Advanced Manufacturing Systems," SAE International Journal of Materials \& Manufacturing, vol. 9, no. 2, pp. 457- 
464, 2016.

[9] J. A. Marvel, J. Falco and I. Marstio, "Characterizing Task-Based HumanRobot Collaboration Safety in Manufacturing," IEEE Transactions on Systems, Man, and Cybernetics: Systems, vol. 45, no. 2, pp. 260-275, 2015.

[10]S. Andreev et al., "Understanding the IoT connectivity landscape: a contemporary M2M radio technology roadmap," IEEE Commun. Mag., vol. 53, no. 9, pp. 32-40, 2015.

[11] Inov360. [Online]. Available: https://www.inov360.com/reseaux-simless-le-nouvel-eldorado-du-m2m-et-de-linternet-des-object-2/. Accessed on: Mar. 20, 2019.

[12] A. Al-Fuqaha, M. Guizani, M. Mohammadi, M. Aledhari, and M. Ayyash, "Internet of Things: A Survey on Enabling Technologies, Protocols, and Applications," IEEE Commun. Surv. Tutorials, vol. 17, no. 4, pp. 23472376, 2015.

[13] S. Gruner, J. Pfrommer, F. Palm, "RESTful Industrial Communication with OPC UA," IEEE Trans. Ind. Informatics, vol. 12, no. 5, pp. 1832-1841, 2016.

[14] J. Wan, B. Chen, M. Imran, F. Tao, D. Li, C. Liu, S. Ahmad, "Toward Dynamic Recource Management for IoT-Based Manufacturing," IEEE Communications Magazine, vol. 56, no. 2, pp. 52-59, 2018.

[15] P. Vrignat, B. Robles, M. Avila, and F. Kratz, "OPC UA: Examples of Digital Reporting Applications for Current Industrial Progresses," In: 2018 Internationa Conference on Electrical, Control, Automation and Robotics, Xiamen, China, Sep. 16-17, 2018. pp. 305-313.

[16] J. Latvakoski et al., "Towards Horizontal Architecture for Autonomic M2M Service Networks," Futur. Internet, vol. 6, no. 2, pp. 261-301, 2014.

[17] J. Davis, T. Edgar, J. Porter, J. Bernaden, and M. Sarli, "Smart Manufacturing, Manufacturing Intelligence and Demand-Dynamic Performance," Comput. Chem. Eng., vol. 47, pp. 145-156, 2012.

[18] J. Swetina, G. Lu, P. Jacobs, F. Ennesser, and J. Song, "Toward a standardized common M2M service layer platform: Introduction to oneM2M," IEEE Wirel. Commun., vol. 21, no. 3, pp. 20-26, 2014.

[19]Microsoft Discrete Group Manufacturing, "A Discrete Manufacturing Reference Architecture (DIRA) Framework Whitepaper," 2011.

[20] M. S. Ali, E. Hossain, and D. I. Kim, "LTE/LTE-A Random Access for Machine-Type Communications in Smart Cities,", IEEE Commun.Mag., vol. 55, no. 1, pp. 76-83, 2017.

[21] H. M. Gursu, M. Vilgelm, W. Kellerer, M. Reisslein, "Hybrid Collision Avoidance-Tree Resolution for MM Random Access," IEEE Trans. Aeros. and Elect. Sys., vol. 53, no. 4, pp. 1974-1987, 2017.

[22]B. Xu, L. Da Xu, H. Cai, C. Xie, J. Hu, and F. Bu, "Ubiquitous data accessing method in iot-based information system for emergency medical services," IEEE Trans. Ind. Informatics, vol. 10, no. 2, pp. 1578-1586, 2014.

[23]C. H. Liu, B. Yang, and T. Liu, "Efficient Naming, Addressing and Profile Services in Internet-of-Things Sensory Environments," Ad Hoc Networks, vol. 18, pp. 85-101, 2014.

[24]Z. Meng, Z. Wu, C. Muvianto, J. Gray, "A Data-Oriented M2M Messaging Mechanism for Industrial IoT Applications," IEEE Internet Things J., vol. 4, no. 1, pp. 236-246, 2017.

[25] H. Cai, L. Xu, B. Xu, C. Xie, S. Qin, and L. Jiang, "IoT-based Configurable Information Service Platform for Product Lifecycle Management," IEEE Trans. Ind. Informatics, vol. PP, no. 2, pp. 1-1, 2014.

[26] J. Kim, J. Lee, J. Kim, and J. Yun, "M2M service platforms: Survey, issues, and enabling technologies," IEEE Commun. Surv. Tutorials, vol. 16, no. 1, pp. 61-76, 2014.

[27]D. T. Meridou et al., "An Ontology-Based Smart Production Management System," IT Prof., vol. 17, no. 6, pp. 36-46, 2015.

[28] M. P. Papazoglou, W. J. Van Den Heuvel, and J. E. Mascolo, "A reference architecture and knowledge-based structures for smart manufacturing networks," IEEE Softw., vol. 32, no. 3, pp. 61-69, 2015.

[29]F. Tao, Y. Cheng, L. Da Xu, L. Zhang, and B. H. Li, "CCIoT-CMfg: Cloud computing and internet of things-based cloud manufacturing service system," IEEE Trans. Ind. Informatics, vol. 10, no. 2, pp. 1435-1442, 2014.

[30] A. Garstenauer, T. Blackburn, B. Olson, "A Knowledge Mangement Based Approach to Quality Management for Large Manufacturing Organizations," Engineering mangement Journal, vol. 26, no. 4, pp.47-58, 2014.

[31]M. Ben Alaya, S. Medjiah, T. Monteil, and K. Drira, "Toward semantic interoperability in oneM2M architecture," IEEE Commun. Mag., vol. 53, no. 12 , pp. 35-41, 2015.

[32] J. Wan, B. Yin, D. Li, A. Celesti, F. Tao, Q. Hua, "An Ontology-Based Resource Reconfiguration Method for Manufacturing Cyber-Physical Systems," IEEE/ASME Transactions on Mechatronics, vol. 23, no. 6, pp.2537-2546, 2018.
[33]I. Grangel-González, L. Halilaj, G. Coskun, S. Auer, D. Collarana, and M. Hofmeister, "Towards a Semantic Administrative Shell for Industry 4.0 Components," arXiv Prepr. arXiv1601.01556, 2016.

[34] European Commission, "Final Report Summary - PICKNPACK (Flexible robotic systems for automated adaptive packaging of fresh and processed food products), $"$ [Online]. Available: https://cordis.europa.eu/project/rcn/105072/reporting/en, Accessed on: Mar. 20, 2019. 